\title{
Modeling the Bistability of Laminated Composite Toroidal Slit Tubes
}

\author{
Geoffrey Knott* Andrew Viquerat ${ }^{\dagger}$ \\ Surrey Space Centre 83 Dept. of Mechanical Engineering Sciences, University of Surrey, UK
}

\begin{abstract}
The bistability of a toroidal slit tube is modeled using the Rayleigh-Ritz method. Approximate explicit expressions for the original stable deployed geometry, and the deformed stowed geometry are used to derive forms for the bending and stretching strain energy. The surface of a torus has varying Gaussian curvature, requiring a new approach to the modeling and analysis of the stable configurations. A comparative study with established straight-BRC models was conducted from which the doubly curved-BRC model presented here predicts second stable state coil radii with $96.25 \%$ agreement.
\end{abstract}

\section{Introduction}

$\mathrm{T}$ HE invention and investigation of bistable reeled composites ${ }^{1}$ (BRCs) has led to many valuable applications in the sectors of security, civil engineering, energy, mining, consumer, defense and aerospace. BRCs are open-section tubular structures that can be rolled up and extended, analogous to tape measures, but are stable in both configurations without the need of support structures. Bistability arises due to the arrangement of fibers in each ply $^{2}$ through an antisymmetric lay-up scheme with respect to the mid-plane which results in a preferential direction for bending to occur ${ }^{3}$ and theoretically may be exhibited using any material of high Poisson ratio and sufficient stiffness ${ }^{4}$.

Various approaches, modeling and analyses of shells ${ }^{5}$ and BRCs over the years include finite element analysis ${ }^{6}$ to predict and characterize the rolled-up configuration and beam models ${ }^{7,8,9}$ to predict second equilibrium position and stability. Progressing to a shell model ${ }^{10}$ enabled the study of edge effects and an inextensional bending model ${ }^{11}$ utilized a property of developable surfaces - the Gaussian curvature ${ }^{12}$ remains zero everywhere - to predict coiled radius by modeling deformation of a plate superimposed upon the surface of a great cylinder.
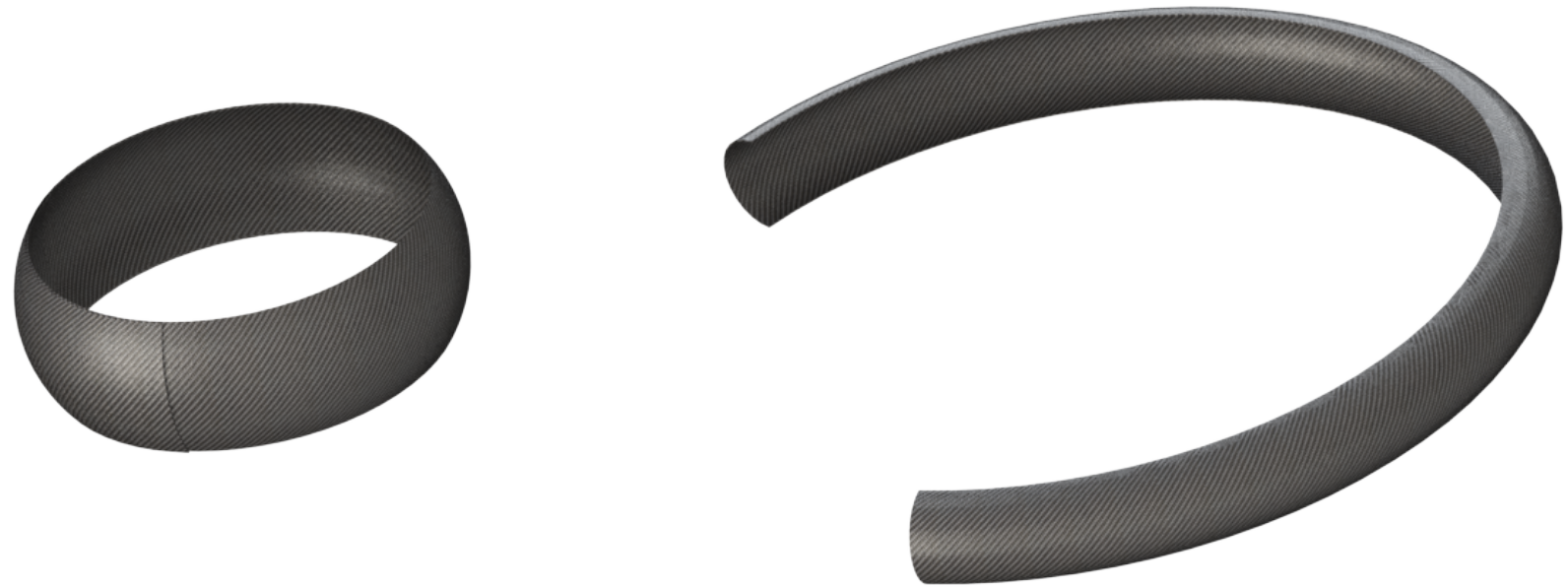

Figure 1: A torus-BRC rendering. Coiled/stowed (left) to deployed/extended (right)

*PhD Candidate

${ }^{\dagger}$ Lecturer 
In this paper, we begin investigating the doubly curved torus-BRC as illustrated in Figure 1 which until now has only been briefly discussed ${ }^{4}$.

\section{A. Geometric Model}

Typically, a complete torus is represented using two radii, $R$ and $r$ to describe major and minor circles respectively as shown in Figure 2. The torus is an example of a surface of revolution whereby the crosssection is swept through an angle about an axis of revolution, resulting in a 3D solid or shell. We consider the point $P$ on the torus surface with longitudinal and transverse principle curvatures $\kappa_{x_{0}}=\frac{1}{R+r}$ and $\kappa_{y_{0}}=\frac{1}{r}$. A particular property of the torus is the Gaussian curvature, $K_{g}=\kappa_{x} \kappa_{y}$ which varies along the cross-section - negative maxima closest to the axis of revolution (at $R-r$ distance), positive maxima at point $P$ and zero along the top and bottom (at $R$ distance).

We define the local, on-surface longitudinal and transverse directions with curvilinear co-ordinate axes $x-y$ respectively, within the global X-Y-Z Cartesian co-ordinate system in which the torus lies with equatorial width $\in(-(R+r), R+r)$ along the $\mathrm{X}$-, Y-axes and height $\in(-r, r)$ along the $\mathrm{Z}$-axis.

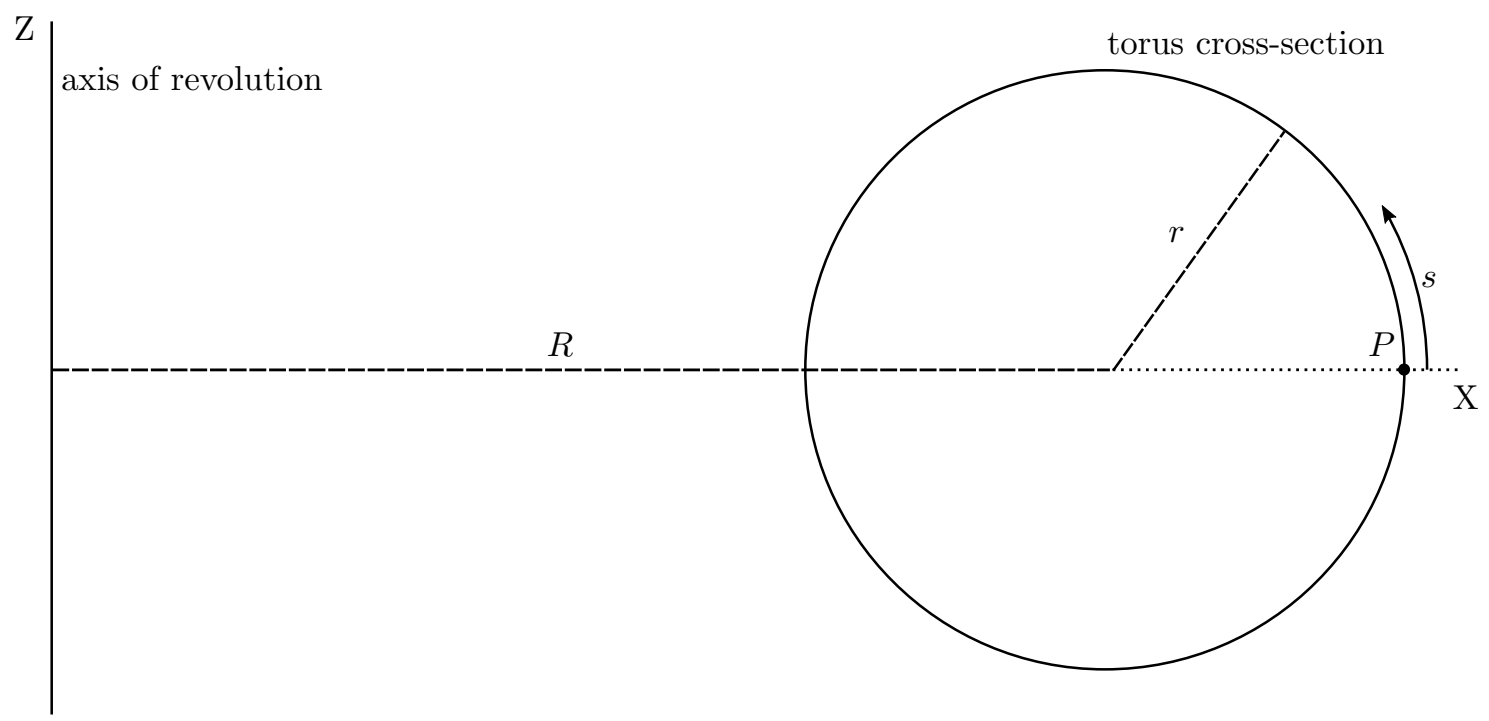

Figure 2: A torus is defined by major $(R)$ and minor $(r)$ circles

The initial, deployed torus cross-section, $\mathbf{T}_{0}(s)$ is modeled as:

$$
\mathbf{T}_{0}(s)=x_{0}(s) \hat{\mathbf{e}}_{X}+z_{0}(s) \hat{\mathbf{e}}_{Z}
$$

where,

$$
\begin{gathered}
x_{0}(s)=\frac{1}{\kappa_{x_{0}}}-\frac{1}{\kappa_{y_{0}}}+\frac{1}{\kappa_{y_{0}}} \cos \left(s \kappa_{y_{0}}\right) \\
z_{0}(s)=\int_{0}^{ \pm s} \sqrt{1-\left(\frac{d x_{0}}{d s}\right)^{2}} \mathrm{~d} s
\end{gathered}
$$

and $s \in\left(-\frac{\beta_{y_{0}}}{2 \kappa_{y_{0}}}, \frac{\beta_{y_{0}}}{2 \kappa_{y_{0}}}\right)$ are points along the cross-section of circumference or width, $w_{0}=\frac{\beta_{y_{0}}}{\kappa_{y_{0}}}$, the transverse subtended angle is $\beta_{y_{0}}$, longitudinal and transverse principle curvatures $\kappa_{x_{0}}$ and $\kappa_{y_{0}}$ and point $P$ is located at $x_{0}(s=0)$.

Simulating deformation of the torus slit tube is achieved using the terms $c_{1}$ and $c_{2}$ to denote change in the major and minor circle radii respectively, shown in Figure 3, enabling use of the Rayleigh-Ritz method. The new geometry, $\mathbf{T}(s)$ can be expressed as:

$$
\mathbf{T}(s)=x(s) \hat{\mathbf{e}}_{X}+z(s) \hat{\mathbf{e}}_{Z}
$$


where,

$$
\begin{gathered}
\mathbf{T}(s)=\left[x_{0}(s)+\Delta x(s)\right] \hat{\mathbf{e}}_{X}+z(s) \hat{\mathbf{e}}_{Z} \\
\Delta x(s)=-\underbrace{c_{1}}_{a}-\underbrace{\frac{1}{\kappa_{y_{0}}} \cos \left(s \kappa_{y_{0}}\right)}_{b}-\underbrace{\frac{c_{2}}{\kappa_{y_{0}}}}_{c}+\underbrace{\frac{1+c_{2}}{\kappa_{y_{0}}} \cos \left(\frac{s \kappa_{y_{0}}}{1+c_{2}}\right)}_{d} \\
z(s)=\int_{0}^{ \pm s} \sqrt{1-\left(\frac{d x}{d s}\right)^{2}} \mathrm{~d} s
\end{gathered}
$$

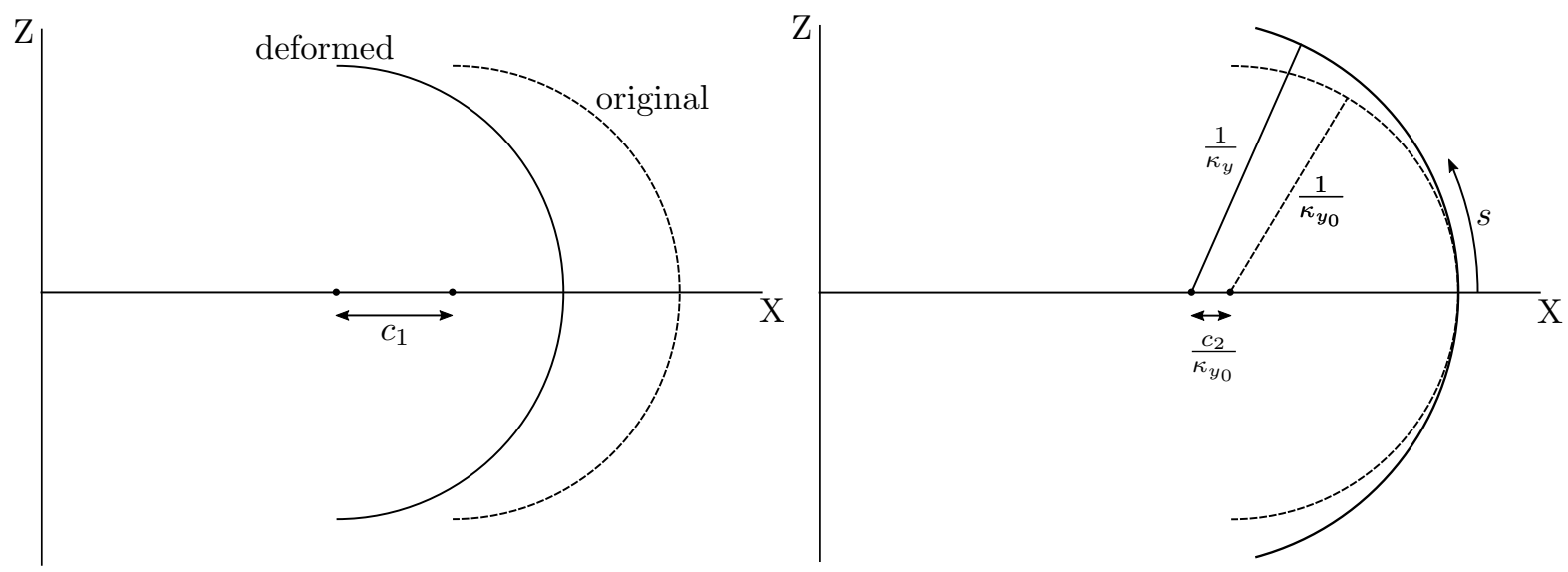

Figure 3: Deformation of the slit tube cross-section due to $c_{1}$ and $c_{2}$

Eq. 6 is constructed such that:

- $a$, defines the X-translation effect of coiling/roll up

- $b$, cancels with the last term in $x_{0}(s)$ from Eq. 2 describing the initial cross-section's X-component in preparation for term $d$

- $c$, negates X-translation of the minor circle's centre-point due to new radius

- $d$, describes the new, deformed, circular cross-section geometry with radius, $\frac{1}{\kappa_{y}}$

When $c_{1}<0$, the torus undergoes stowage/coiling, shown in Table $1 . c_{2}$ is the most effective term deforming the geometry with respect to principle curvatures; when $0<c_{2}$, both the longitudinal and transverse curvature change and describes flattening of the torus cross-section. The effects of $c_{1}$ and $c_{2}$ are also illustrated in Figure $4-\beta_{x}$ describes longitudinal stretching and will be made use of later in Eq. 21 for stretching strain energy calculation.

\begin{tabular}{c|cc} 
& $c_{1}<0$ & $c_{1}=0$ \\
\hline$c_{2}=0$ & roll-up & no change \\
$0<c_{2}$ & flatten and roll-up & flatten
\end{tabular}

Table 1: Deformation effects of $c_{1}$ and $c_{2}$ terms

Given Eqs. 2, 6 and 7, Eq. 5 may be presented as,

$$
\mathbf{T}(s)=\left[\begin{array}{c}
x(s) \\
0 \\
z(s)
\end{array}\right]=\left[\begin{array}{c}
\frac{1}{\kappa_{x_{0}}}-\frac{1}{\kappa_{y_{0}}}-c_{1}-\frac{c_{2}}{\kappa_{y_{0}}}+\frac{1+c_{2}}{\kappa_{y_{0}}} \cos \left(\frac{s \kappa_{y_{0}}}{1+c_{2}}\right) \\
0 \\
\int_{0}^{ \pm s} \sqrt{1-\left(\frac{d x}{d s}\right)^{2}} \mathrm{~d} s
\end{array}\right]
$$




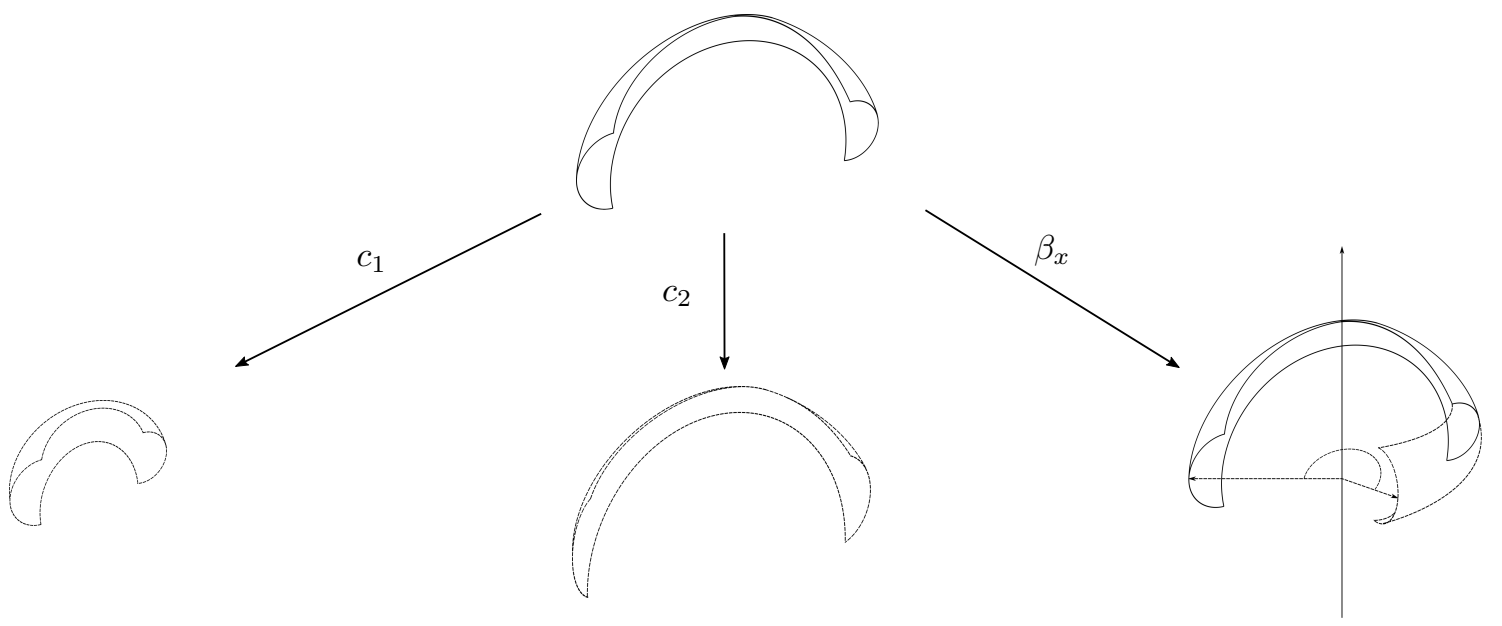

Figure 4: Effects of the deformations $c_{1}, c_{2}$ and $\beta_{x}$

\section{B. Principle Curvatures Calculation}

The longitudinal curvature ${ }^{5}, \kappa_{x}(s)$ varies along the cross-section as seen in Figure 5 . The longitudinal radius of curvature, $d(s)$ is defined as a straight line through the minor circle center, intersecting a point on the cross-section and axis of revolution. $d(s) \perp d s$ over the cross-section. The transverse curvature, $\kappa_{y}$ does not vary with arc-length, $s$ and therefore describes the arc of a great circle.

$$
\kappa_{x}(s)=\frac{1}{d(s)}, \quad \kappa_{y}=\frac{\left|\mathbf{T}^{\prime}(s) \times \mathbf{T}^{\prime \prime}(s)\right|}{\left|\mathbf{T}^{\prime}(s)\right|^{3}}
$$

where,

$$
d(s)=\frac{x(s)}{\cos (\alpha)}
$$

We consider the curvature at each point along the cross-section and follow a geometric approach inset in Figure 5 for an arbitrary point $Q$.

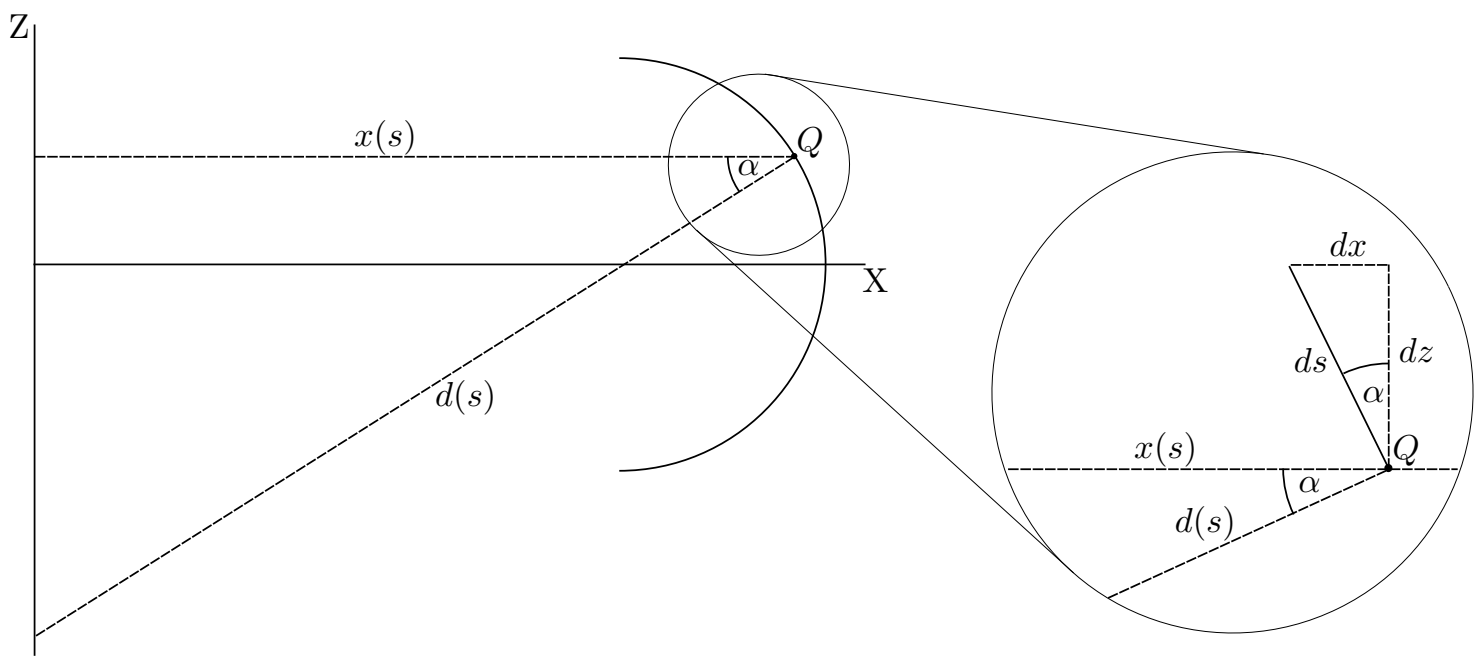

Figure 5: Definition for the longitudinal radius of curvature, $d(s)$ with the elements at each surface point an arc-distance, $s$ from the $\mathrm{X}$-axis 
where,

$$
\alpha=\arcsin \left(\frac{d x}{d s}\right)
$$

and substituting into Eq. 10 yields,

$$
d(s)=\frac{x(s)}{\cos \left(\arcsin \left(\frac{d x(s)}{d s}\right)\right)}=\frac{x(s)}{\sqrt{1-\left(\frac{d x(s)}{d s}\right)^{2}}}
$$

Given Eq. 12, $\kappa_{x}(s)$ from Eq. 9 simply becomes:

$$
\kappa_{x}(s)=\frac{\sqrt{1-\left(\frac{d x(s)}{d s}\right)^{2}}}{x(s)}
$$

Similarly for the initial longitudinal curvature:

$$
\kappa_{x_{0}}(s)=\frac{\sqrt{1-\left(\frac{d x_{0}(s)}{d s}\right)^{2}}}{x_{0}(s)}
$$

$\kappa_{y}$ from Eq. 9 is determined using Eq. 8. It follows that $\kappa_{y}$ may be expressed as,

$$
\kappa_{y}= \pm \frac{\frac{d^{2} x(s)}{d s^{2}}}{\sqrt{1-\left(\frac{d x(s)}{d s}\right)^{2}}}
$$

It can be seen that the Gaussian curvature is positive along the outermost cross-section edge (when $\frac{d x(s)}{d s}<0$ ) and negative along the innermost edge (when $0<\frac{d x(s)}{d s}$ ) given the expressions for longitudinal curvature from Eqs. 13 and 14. The transverse curvature may be positive or negative as a result of the mathematical procedure in Eq. 15, but shall remain positive over all deformations modeled given that the cross-section converges to flattening and no further.

\section{Torus Strain Energy Model: Bending \& Stretching Strain}

The strain energy model used here extends past models ${ }^{1}$, to take into account variable curvature and stretching along the cross-section of the tube. The bending and stretching energy per unit area ${ }^{13}$ are defined as,

$$
\begin{gathered}
u_{b}(s)=\frac{1}{2}\left[\begin{array}{lll}
\Delta \kappa_{x}(s) & \Delta \kappa_{y} & \Delta \kappa_{x y}(s)
\end{array}\right][\mathbf{D}]\left[\begin{array}{c}
\Delta \kappa_{x}(s) \\
\Delta \kappa_{y} \\
\Delta \kappa_{x y}(s)
\end{array}\right] \\
u_{s}(s)=\frac{1}{2}\left[\begin{array}{lll}
\epsilon_{x}(s) & \epsilon_{y} & \epsilon_{x y}(s)
\end{array}\right][\mathbf{A}]\left[\begin{array}{c}
\epsilon_{x}(s) \\
\epsilon_{y} \\
\epsilon_{x y}(s)
\end{array}\right]
\end{gathered}
$$

where change in principle curvature, $\Delta \kappa$ and stretching, $\epsilon_{x}$ are defined respectively as,

$$
\begin{gathered}
\Delta \kappa=\kappa_{\text {deformed }}-\kappa_{\text {initial }} \\
=\kappa-\kappa_{0} \\
\epsilon_{x}(s)=\frac{l_{\text {deformed }}-l_{\text {initial }}}{l_{\text {initial }}}=\frac{l(s)-l_{0}(s)}{l_{0}(s)} \\
5 \text { of } 13
\end{gathered}
$$


where,

$$
\begin{gathered}
l_{0}(s)=\beta_{x_{0}} x_{0}(s) \\
l(s)=\beta_{x} x(s) \\
w_{0}=\beta_{y_{0}} R_{y_{0}}
\end{gathered}
$$

are the initial lengths, $l_{0}(s)$ and deformed lengths, $l(s)$ of imaginary parallel longitudinal "fibers" and initial cross-section circumference, $w_{0}$ (which remains constant due to transverse stretching, $\epsilon_{y}$ being neglected). $\beta_{x_{0}}$ is the initial longitudinal subtended angle and $\beta_{x}$ is the deformed longitudinal subtended angle generally resulting in longitudinal stretching, illustrated in Figure 4.

It follows that changes in longitudinal and transverse curvature are:

$$
\begin{aligned}
\Delta \kappa_{x}(s) & =\frac{\sqrt{1-\left(\frac{d x(s)}{d s}\right)^{2}}}{x(s)}-\frac{\sqrt{1-\left(\frac{d x_{0}(s)}{d s}\right)^{2}}}{x_{0}(s)} \\
\Delta \kappa_{y} & =\frac{\frac{d^{2} x(s)}{d s^{2}}}{\sqrt{1-\left(\frac{d x(s)}{d s}\right)^{2}}}-\frac{1}{R_{y_{0}}}
\end{aligned}
$$

Given $\kappa_{x y}=\epsilon_{x y}=0, D_{13}=D_{23}=0$ and the plate has initial curvature $\kappa_{x_{0}}(s)$ and $\kappa_{y_{0}}$, the bending energy per unit area from Eq. 16 becomes:

$$
\begin{aligned}
& u_{b}(s)=\frac{1}{2}\left[D_{11} \Delta \kappa_{x}^{2}(s)+2 D_{12} \Delta \kappa_{x}(s) \Delta \kappa_{y}+D_{22} \Delta \kappa_{y}^{2}\right] \\
& =\frac{1}{2}\left[D_{11}\left[\kappa_{x}(s)-\kappa_{x_{0}}(s)\right]^{2}\right. \\
& +2 D_{12}\left[\kappa_{x}(s)-\kappa_{x_{0}}(s)\right]\left[\kappa_{y}-\kappa_{y_{0}}\right] \\
& \left.+D_{22}\left[\kappa_{y}-\kappa_{y_{0}}\right]^{2}\right]
\end{aligned}
$$

and given Eqs. 19, 20 and 21, the stretching energy per unit area from Eq. 17 becomes:

$$
\begin{aligned}
u_{s}(s) & =\frac{1}{2} A_{11} \epsilon_{x}^{2}(s) \\
& =\frac{1}{2} A_{11}\left[\frac{l(s)-l_{0}(s)}{l_{0}(s)}\right]^{2}
\end{aligned}
$$

The bending energy per unit length is:

$$
\begin{aligned}
U_{b} & =\iint_{A_{0}} u_{b}(s) \mathrm{d} A \\
& =2 \int_{0}^{l_{0}} \int_{0}^{\frac{w_{0}}{2}} u_{b}(s) \mathrm{d} s \mathrm{~d} l \\
& =2 \int_{0}^{\frac{w_{0}}{2}} u_{b}(s) \cdot l_{0}(s) \mathrm{d} s
\end{aligned}
$$

Similarly, the stretching energy per unit length is:

$$
\begin{aligned}
U_{s} & =\iint_{A} u_{s}(s) \mathrm{d} A \\
& =2 \int_{0}^{l} \int_{0}^{\frac{w_{0}}{2}} u_{s}(s) \mathrm{d} s \mathrm{~d} l \\
& =2 \int_{0}^{\frac{w_{0}}{2}} u_{s}(s) \cdot l(s) \mathrm{d} s
\end{aligned}
$$


The integral limits for bending (Eq. 27) and stretching energy (Eq. 28) differ because stretching is not considered for the bending energy, therefore integration is executed over the initial area, $A_{0}=2 \int_{0}^{l_{0}} \int_{0}^{\frac{w_{0}}{2}} d s d l$.

Locally stable configurations are defined as,

$$
\left[\frac{d}{d c_{1}}, \frac{d}{d c_{2}}, \frac{d}{d \beta_{x}}\right] U_{t}=0
$$

where $U_{t}=U_{b}+U_{s}$ is the total strain energy.

Figure 6 illustrates the approach presented beginning with a deployed torus, $\mathbf{T}_{0}(s)$ where induced deformations $c_{1}$ and $c_{2}$ result in a deformed torus geometry $\mathbf{T}(s)$. The initial surface curvature is then subtracted from the deformed surface curvature to determine the change in curvature at every point along the crosssection, $\Delta \kappa(s)$ and bending energy per unit area, $u_{b}(s)$. The strain is calculated using the initial and deformed tube lengths including longitudinal stretching due to $\beta_{x}$ - leading to the stretching energy per unit area, $u_{s}(s)$. The bending and stretching energy per unit area are then integrated across the surface area of the deformed torus and summed together to find the total strain energy per unit length, $U_{t}\left(c_{1}, c_{2}, \beta_{x}\right)$ - the total strain energy has units $\mathrm{Jm}^{-1}$ due to the surface integral limits for the tube length being zero to unity $(1 \mathrm{~m})$. The total strain energy is then plotted in order to find local minima at which point corresponds to a specific set of values for $c_{1}, c_{2}$ and $\beta_{x}$ which describe the second stable state geometry.

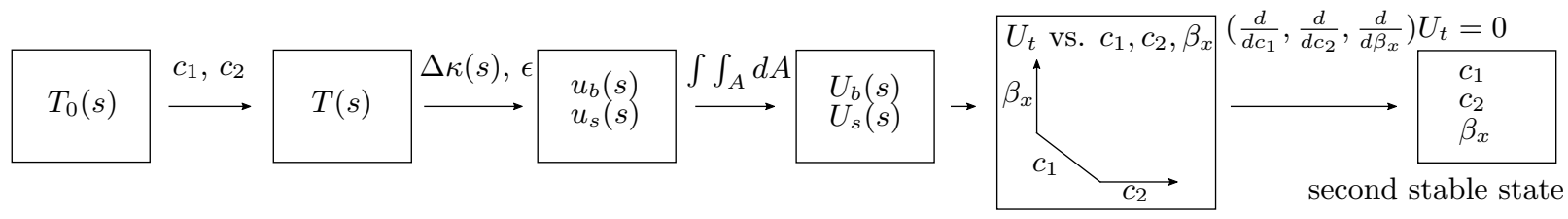

Figure 6: Flow chart of the method presented

\section{Results}

The modeled laminated composite toroidal slit tube has unidirectional ply properties in Table 2 .

\begin{tabular}{c|c|c} 
Property & Value & Units \\
\hline Fiber modulus & $240 \times 10^{9}$ & $\mathrm{~Pa}$ \\
Fiber shear modulus & $95 \times 10^{7}$ & $\mathrm{~Pa}$ \\
Fiber Poisson ratio & 0.22 & - \\
Matrix modulus & $4 \times 10^{9}$ & $\mathrm{~Pa}$ \\
Matrix shear modulus & $2.7 \times 10^{9}$ & $\mathrm{~Pa}$ \\
Matrix Poisson ratio & 0.35 & - \\
Fiber volume fraction & $\frac{1}{2}$ & - \\
Unidirectional layer thickness & $0.213 \times 10^{-3}$ & $\mathrm{~m}$ \\
Number of plies & 5 & -
\end{tabular}

Table 2: Unidirectional ply mechanical properties

With ABD-matrix for an anti-symmetric $45^{\circ}$ layup, $\left[+45^{\circ},-45^{\circ}, 90^{\circ},+45^{\circ},-45^{\circ}\right]$ calculated $^{1,2,4,11}$ :

$$
\left[\begin{array}{cc}
\mathbf{A} & \mathbf{B} \\
\mathbf{B} & \mathbf{D}
\end{array}\right]=\left[\begin{array}{ccc|ccc}
3.49 \times 10^{7} & 2.48 \times 10^{7} & 0 & 0 & 0 & -2.60 \times 10^{3} \\
2.48 \times 10^{7} & 5.94 \times 10^{7} & 0 & 0 & 0 & -2.60 \times 10^{3} \\
0 & 0 & 2.80 \times 10^{7} & -2.60 \times 10^{3} & -2.60 \times 10^{3} & 0 \\
\hline 0 & 0 & -2.60 \times 10^{3} & 3.90 & 2.85 & 0 \\
0 & 0 & -2.60 \times 10^{3} & 2.85 & 3.99 & 0 \\
-2.60 \times 10^{3} & -2.60 \times 10^{3} & 0 & 0 & 0 & 3.15
\end{array}\right]
$$

where $\mathbf{A}, \mathbf{B}$ and $\mathbf{D}$ have units $\mathrm{Pa} \mathrm{m}, \mathrm{Pa} \mathrm{m}^{2}$ and $\mathrm{Pa} \mathrm{m}^{3}$ respectively. 


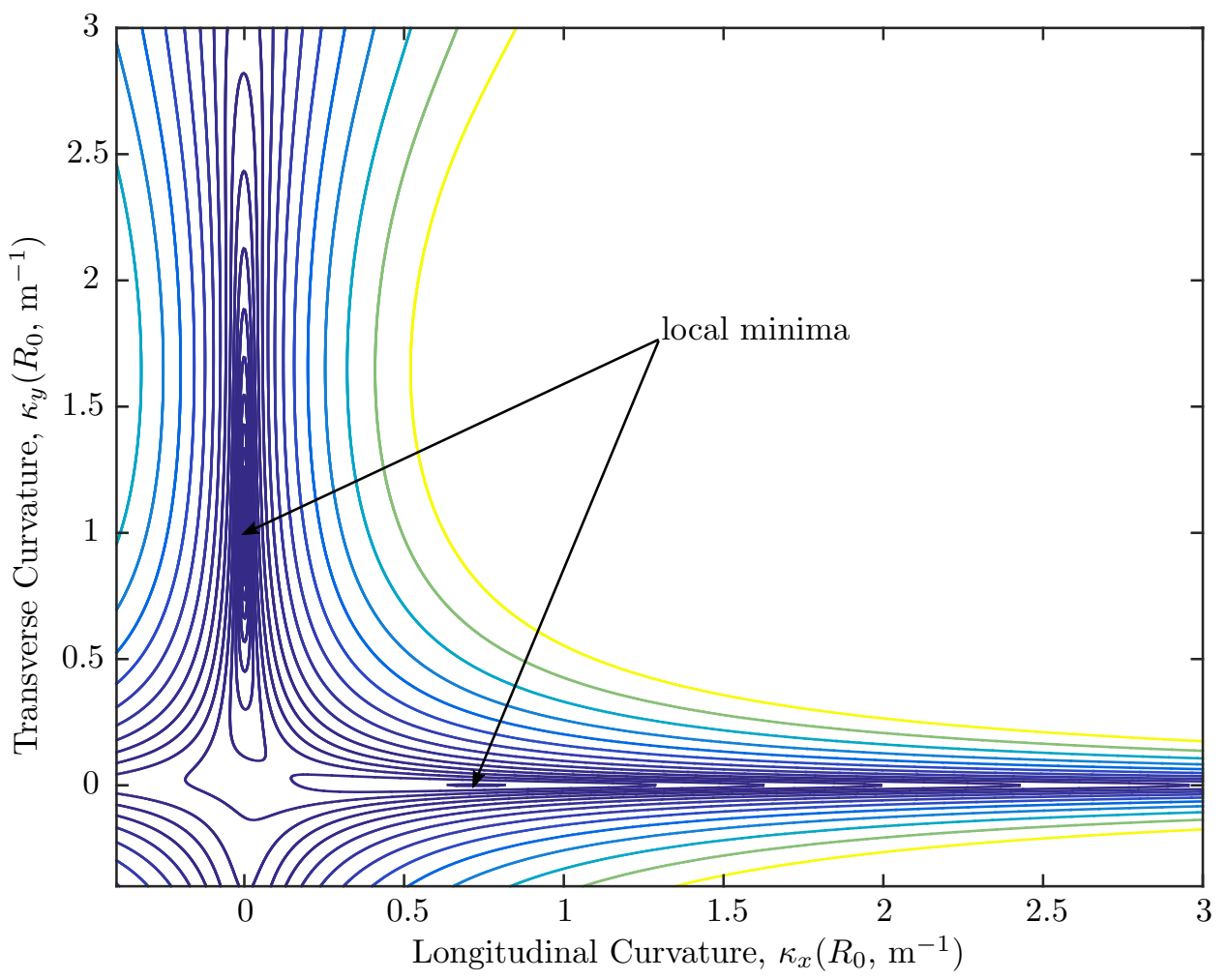

Figure 7: Strain energy contour plot using Iqbal's model. ${ }^{1}$ The curvature is in terms of $R_{0}$

\section{A. Straight-BRC Model}

The straight-BRC modeled has initial geometry of $R_{y_{0}}=3.5 \mathrm{~cm}$ and $\beta_{y_{0}}=\pi$. Figure 7 predicts two stable states at $\kappa_{x_{0}}=0, \kappa_{y_{0}}=1$ and $\kappa_{x} \approx 0.73, \kappa_{y}=0$. This equates to a predicted coil radius of approximately $2.56 \mathrm{~cm}$ and for a BRC of length $1 \mathrm{~m}$, the coil subtended angle is approximately 39 radians or 6.2 turns. This value, $\beta_{x} \approx 39^{c}$ narrows the search for where the second stable point is expected to be in the curved-BRC model results.

\section{B. Doubly Curved-BRC Model}

To validate the doubly curved model, toruses with very small longitudinal curvature were compared to straight-BRCs. Predictions for the coil radii of straight- and shallowly doubly curved-BRCs are presented in Table 3. For the curved-BRC modeling results, initial longitudinal curvature was gradually decreased in order to investigate the hypothesized convergence to the straight-BRC coil radius result.

The most precise result in Table $3,2.66 \mathrm{~cm}$ for Curved- (5) compares very well to the Straight-BRC prediction of $2.56 \mathrm{~cm}$ and lies within approximately $3.75 \%$.

\begin{tabular}{c|c|c|c|c|c} 
BRC Model & $\kappa_{x_{0}}\left(\mathrm{~m}^{-1}\right)$ & $R_{x}(\mathrm{~cm})$ & $l(\mathrm{~m})$ & $\beta_{x}($ radians $)$ & $\kappa_{y}\left(\mathrm{~m}^{-1}\right)$ \\
\hline Straight- & 0 & $2.56 \pm 0.32$ & 1 & $39.74 \pm 4.9$ & 0 \\
Curved- (1) & 0.3142 & $2.38 \pm 0.13$ & 1.0061 & $\approx 28.57$ & less than 0.468 \\
Curved- (2) & 0.1571 & $2.38 \pm 0.13$ & 1.0004 & $\approx 28.57$ & less than 0.468 \\
Curved- (3) & 0.0393 & $2.62 \pm 0.08$ & 1.0069 & $\approx 38.42$ & less than 0.468 \\
Curved- (4) & 0.0196 & $2.62 \pm 0.08$ & 1.0069 & $\approx 38.42$ & less than 0.468 \\
Curved- (5) & 0.0196 & $2.66 \pm 0.02$ & 1.0062 & $37.90 \pm 1.78$ & less than 0.468
\end{tabular}

Table 3: BRC model prediction comparison for the second stable state coil radius of a $1 \mathrm{~m}$ long tube with cross-section radius $3.5 \mathrm{~cm}$ and subtended angle $\pi$ 
The total strain energy, $U_{t}=U_{b}\left(c_{1}, c_{2}\right)+U_{s}\left(c_{1}, c_{2}, \beta_{x}\right)$ is presented in Figure 8 and plotted with respect to independent variables $c_{1}, c_{2}$ and $\beta_{x}$.

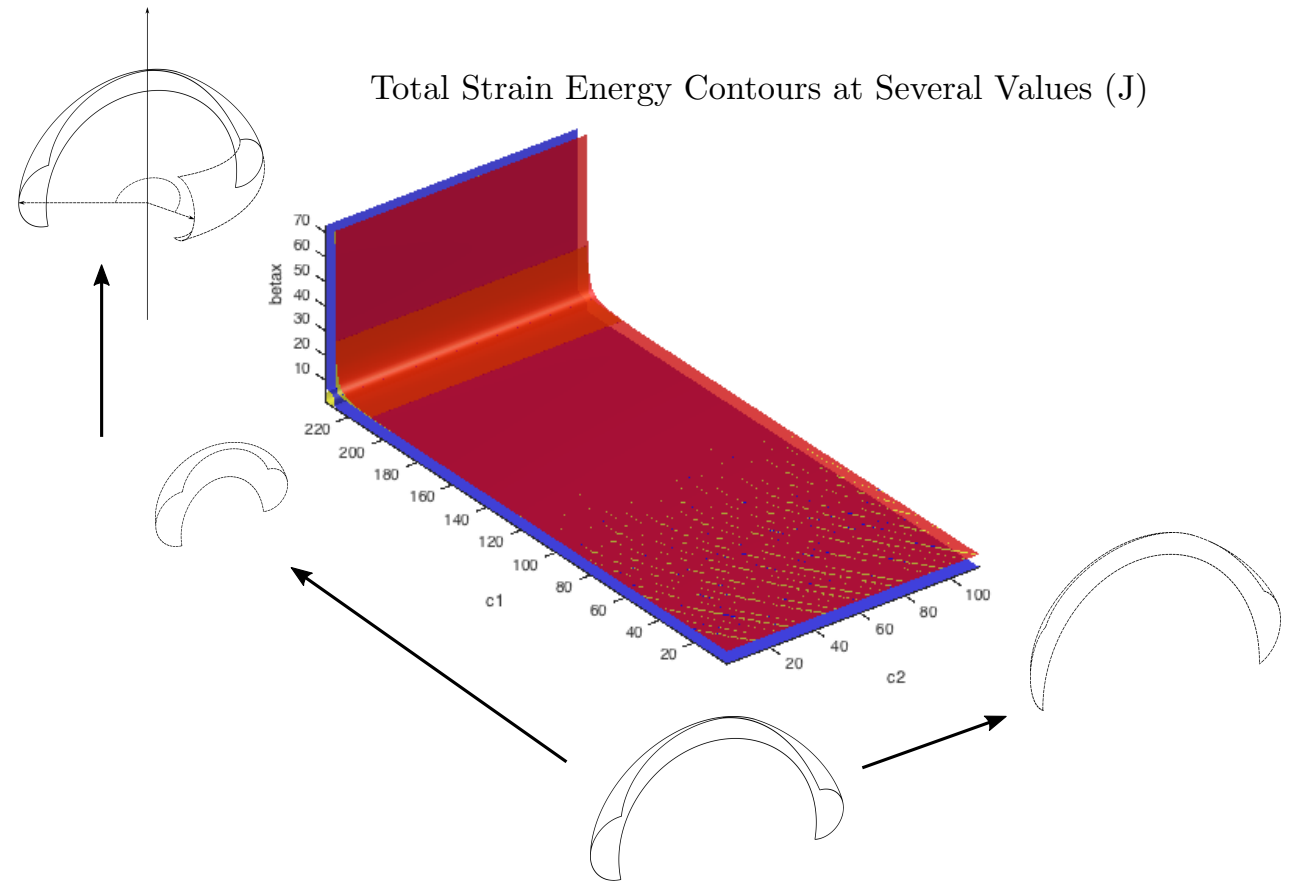

Figure 8: Total strain energy surface plot over all deformations through $c_{1}-c_{2}-\beta_{x}$ space. $\left(0 \leq c_{1} \leq\left(R_{x_{0}}-\right.\right.$ $0.001 \mathrm{~m}), 0 \leq c_{2} \leq 60$ and $\beta_{x_{0}} \leq \beta_{x} \leq 100^{c}$ where $R_{x_{0}} \approx 25.4 \mathrm{~m}$ and $\beta_{x_{0}}=\pi$
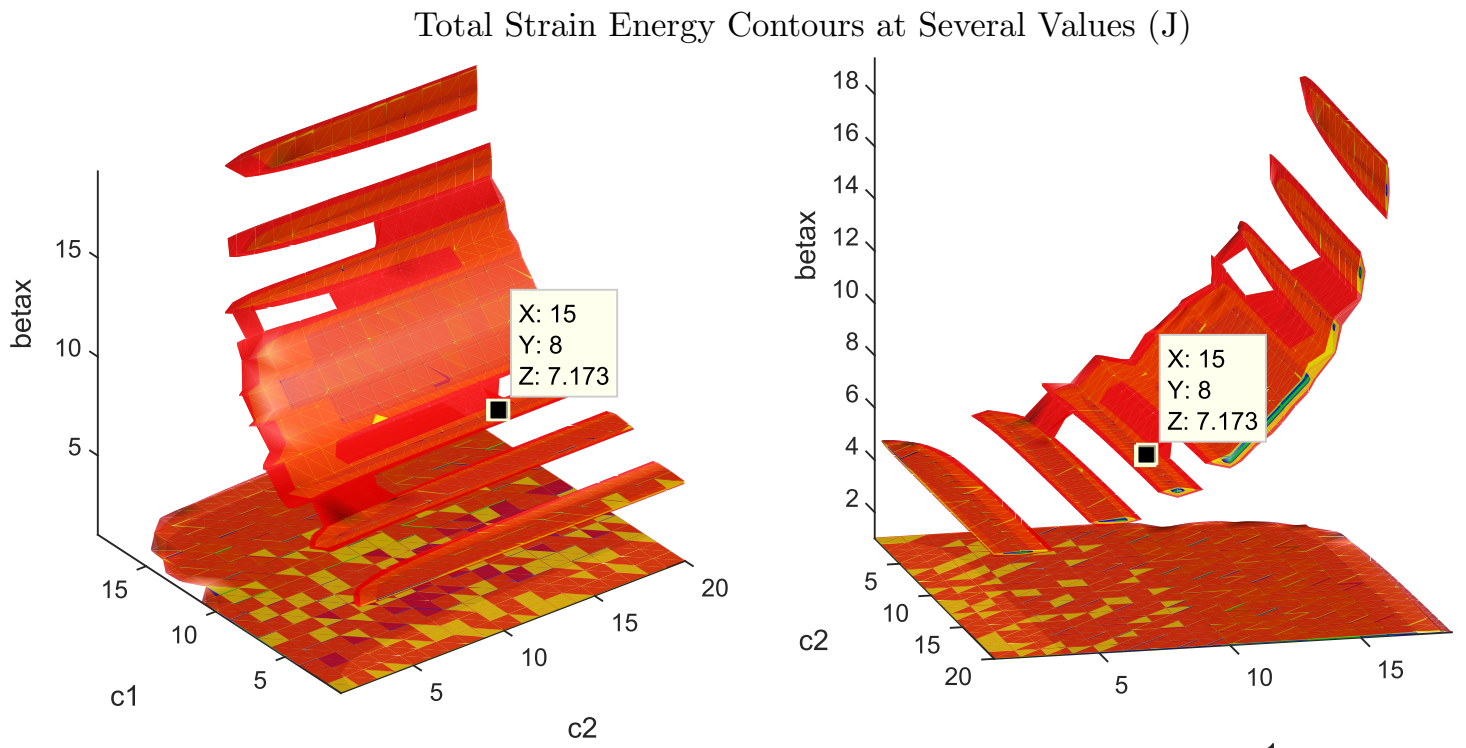

Figure 9: A more detailed analysis of the strain energy surface plot from Figure 8 in the region; $\left(R_{x_{0}}-0.05 \mathrm{~m}\right)$ $\leq c_{1} \leq\left(R_{x_{0}}-0.001 \mathrm{~m}\right), 0 \leq c_{2} \leq 60$ and $\beta_{x_{0}} \leq \beta_{x} \leq 100^{c}$ where $R_{x_{0}} \approx 25.4 \mathrm{~m}$ and $\beta_{x_{0}}=\pi$. Strain energy surface plot showing a low energy channel appearing to form a qualitative $c_{1}=\beta_{x}^{2}$-path and from a different perspective (right), the plot showing the low energy channel continues through $c_{2}$ 

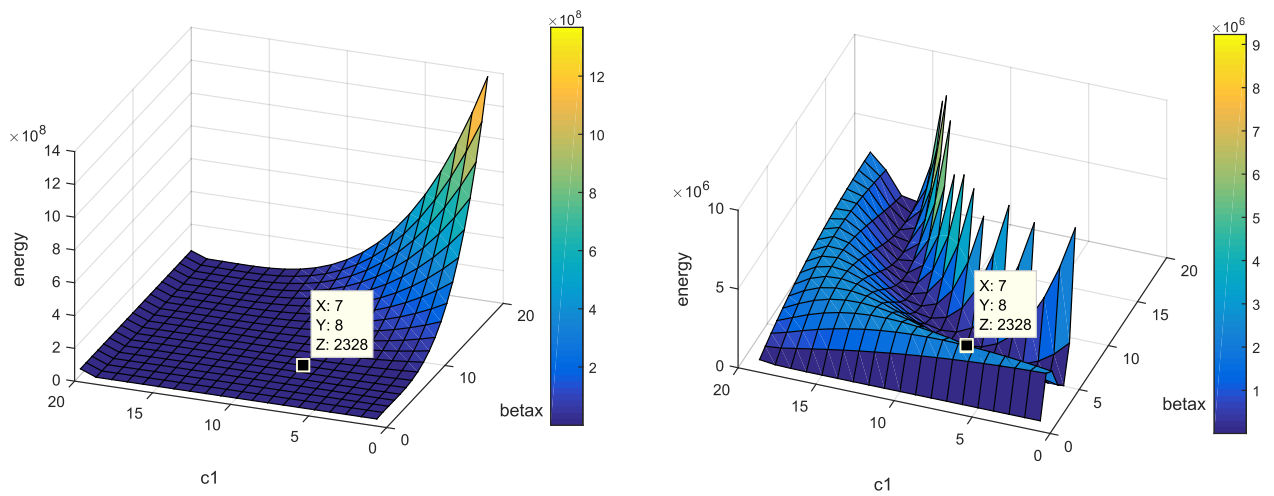

Figure 10: Energy surface plots against $\beta_{x^{-}} c_{1}$ at $c_{2}=15$ from Figure 9. The maximum energy is limited to $10^{9} \mathrm{Jm}^{-1}$ (left) and the energy valley is easily observed now the maximum energy is limited to $10^{7} \mathrm{Jm}^{-1}$ (right)

Modeling of highly curved toroidal-BRCs has been conducted and predicts bistability with the second stable state coil radii presented in Table 4 and Figure 11 - coil radius predictions for toroidal-BRCs with ascending initial longitudinal curvature. All of the toroidal-BRCs modeled have initial longitudinal centerline "fiber" length, $l_{0}(s=0)=1 \mathrm{~m}$ transverse radius of curvature, $R_{y_{0}}=3.5 \mathrm{~cm}$ and transverse subtended angle, $\beta_{y_{0}}=\pi$.

\begin{tabular}{c|c|c|c|c|}
$\beta_{x_{0}}$ (radians) & $\kappa_{x_{0}}\left(\mathrm{~m}^{-1}\right)$ & $R_{x} \pm 0.07(\mathrm{~cm})$ & $\beta_{x} \pm 0.63$ (radians) & $\kappa_{y} \pm 0.33\left(\mathrm{~m}^{-1}\right)$ \\
\hline$\frac{\pi}{4}$ & 0.79 & 5.84 & 17.16 & 1.36 \\
$\frac{\pi}{3}$ & 1.05 & 5.43 & 18.42 & 1.36 \\
$\frac{\pi}{2}$ & 1.57 & 4.78 & 20.79 & 1.36 \\
$\frac{3 \pi}{4}$ & 2.36 & 2.84 & 34.84 & 1.36 \\
$\pi$ & 3.14 & 2.64 & 37.47 & 1.36 \\
$\frac{5 \pi}{4}$ & 3.93 & 2.29 & 42.90 & 1.36 \\
$\frac{3 \pi}{2}$ & 4.71 & 2.10 & 45.90 & 1.36 \\
$\frac{7 \pi}{4}$ & 5.50 & 1.60 & 60.26 & 1.36
\end{tabular}

Table 4: The predicted second stable state coil radii for toroidal-BRCs with ascending initial longitudinal curvature

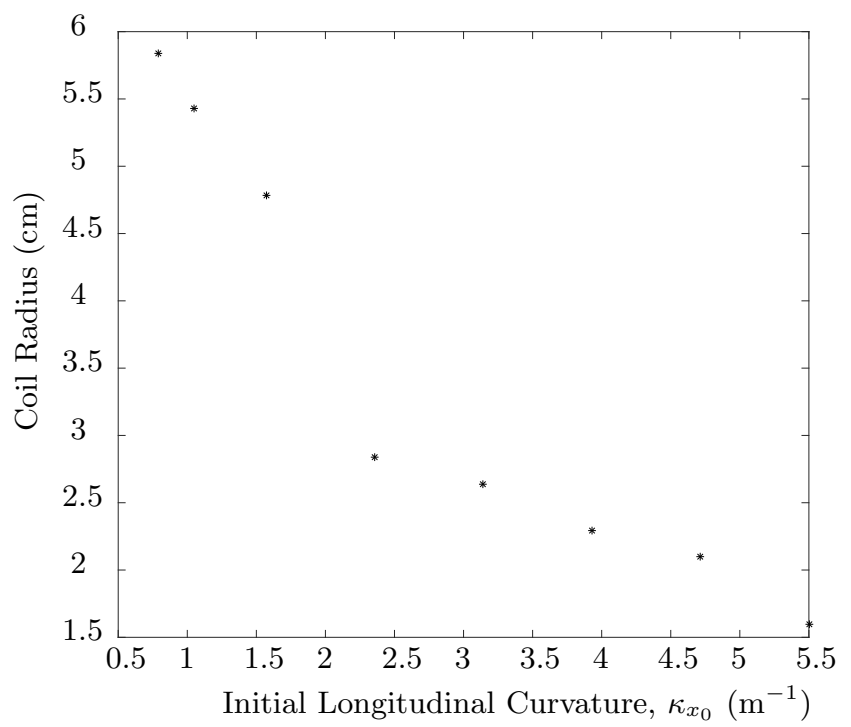

Figure 11: The predicted second stable state coil radius versus initial longitudinal curvature for toroidalBRCs 

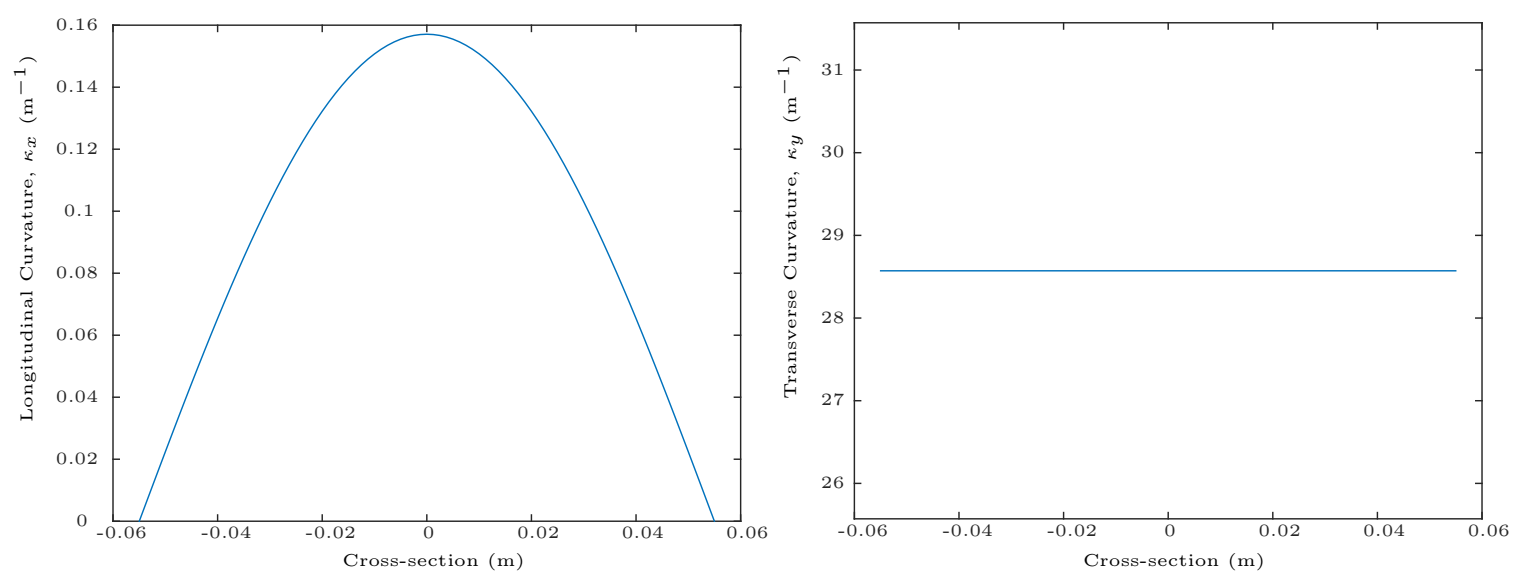

Figure 12: Initial longitudinal curvature, $\kappa_{x_{0}}(s)$ vs. cross-section, $s$ (left) and initial transverse curvature, $\kappa_{y_{0}}$ vs. cross-section, $s$ (right)

\section{The Geometric Model}

Modeling doubly curved-BRCs with initially non-zero transverse and longitudinal curvature and subtended angle has been performed with a verification presented in Figure 12. Figure 12 (left) presents the initial longitudinal curvature, $\kappa_{x_{0}}$ along the cross-section of a fully deployed i.e. stable toroidal-BRC. As expected, $\kappa_{x_{0}}$ varies between extrema of zero along the edges and $\left(\frac{1}{R_{x_{0}}+R_{y_{0}}}\right)$ along the centerline of the BRC. Figure 12 (right) presents the uniform transverse curvature across the circular cross-section.

Figure 13 illustrates the effects of simulating deformation, $c_{1}$ and $c_{2}$ on the longitudinal and transverse curvatures. Top-left in the figure simulates the effect of rolling up only on the longitudinal curvature, when $c_{1}<0, c_{2}=0$. Top-right in the figure shows the independence between only rolling up and the transverse curvature. Bottom-left in the figure simulates the effect of flattening only on the longitudinal curvature, where $c_{1}=0$ and $0<c_{2}$. Bottom-right in the figure simulates flattening only and its effect on transverse curvature.

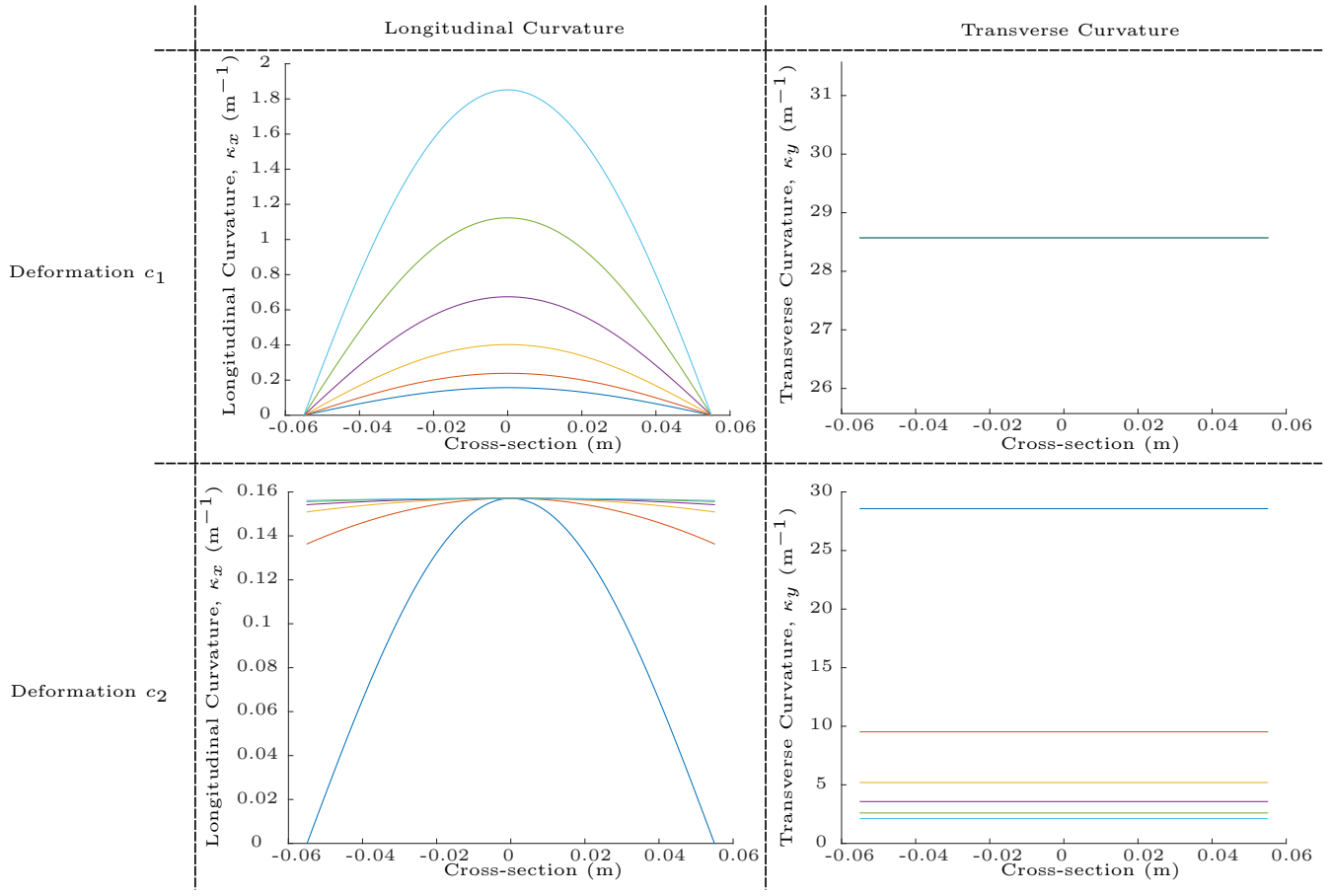

Figure 13: Longitudinal and transverse curvature plotted against cross-section after deformation due to $c_{1}$ and $c_{2}$; top-left) $\kappa_{x}(s)$ for $c_{1}$ top-right) $\kappa_{y}$ for $c_{1}$ bottom-left) $\kappa_{x}(s)$ for $c_{2}$ and bottom-right) $\kappa_{y}$ for $c_{2}$ 


\section{Modeling Bistability with Non-Uniform Transverse Curvature}

Removing the assumption of uniform transverse curvature is achieved by adding higher frequency cosine terms in Eq. 6 - higher order polynomial terms are appropriate also. As a result, the second stable state cross-section of several toroidal-BRCs with various initial longitudinal radii of curvature, $R$ ABD-matrix in Eq. 30, minor radius $R_{y_{0}}=1.6 \mathrm{~cm}$, transverse subtended angle, $\beta_{y_{0}}=160^{\circ}$ and thirteen parameters (including $c_{1}, c_{2}$ and $\beta_{x}$ ) are modeled and presented in Figure 14.

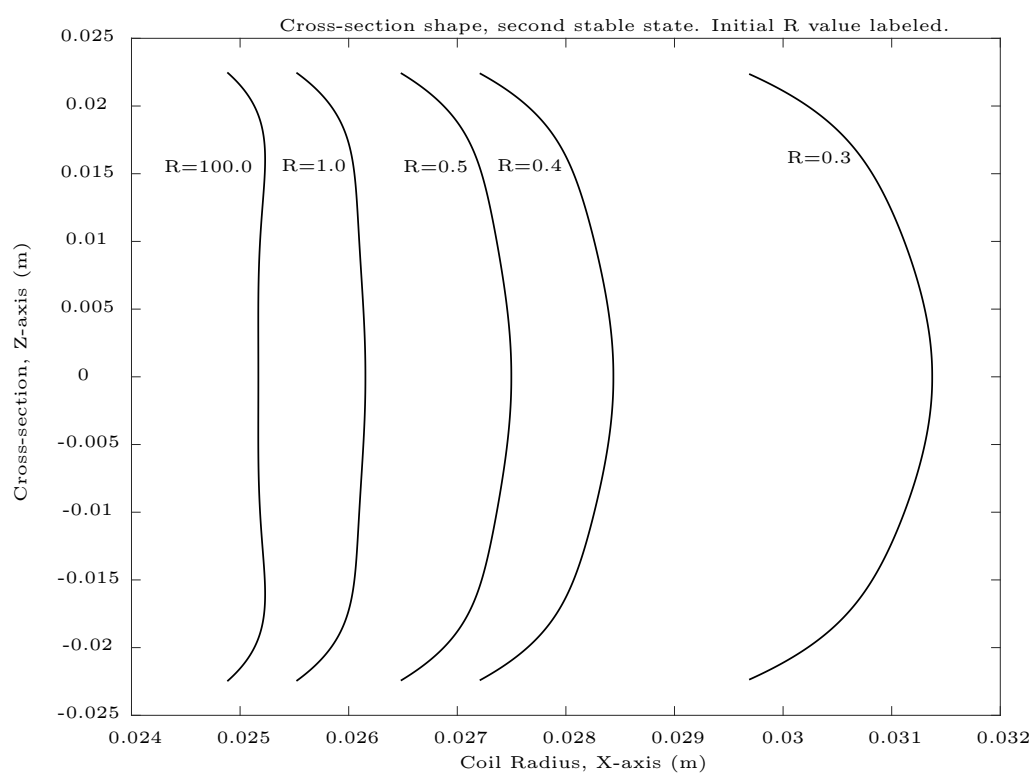

Figure 14: The modeled doubly curved-BRC cross-section for several values of initial longitudinal radius of curvature, $R(\mathrm{~m})$ illustrating the physical result of modeling non-uniform transverse curvature

\section{Discussion}

The method used in this paper is presented in Section I and outlines a mathematical approach treating doubly curved-BRCs as a torus. This approach allows the modeling of non-developable surfaces (where $K_{g} \neq 0$ ), surfaces with variable Gaussian curvature and specifically BRCs with initially non-zero longitudinal and transverse curvature. Given that this approach considers surfaces with variable Gaussian curvature, computation is akin to finite element modeling and requires long computation times to produce results with good accuracy and precision.

In order to verify the doubly curved-BRC model developed, a comparison with other existing models for straight-BRC predictions for the second stable state is performed and presented in Table 3. This is achieved with the curved-BRC model by setting the initial longitudinal curvature, $\kappa_{x_{0}}$ to be very small i.e. very large radius of curvature, $R_{x_{0}}$ and very small longitudinal subtended angle, $\beta_{x_{0}}$. Using this approach, it is hypothesized that the curved-BRC model will converge to approximately predict the second stable state for a straight-BRC.

The effects of $c_{1}$ and $c_{2}$ on the geometry of the BRC have been established as rolling up and flattening respectively (Table 1 ). The effect of changing $\beta_{x}$ is to alter the longitudinal subtended angle and therefore the length of the BRC consequently inducing longitudinal stretching, $\epsilon_{x}$.

In Figure 8, there are regions for which certain values of $c_{1}, c_{2}$ and $\beta_{x}$ equate to relatively low strain energy - enclosed by blue surface contours with yellow and red indicating ascending strain energy surfaces. A "slab" of relatively low energy extends across $c_{2}$ and $\beta_{x}$ at very high $c_{1}$ - this region corresponds to geometry resembling a coil.

A more precise investigate of the aforementioned region is shown in Figure 9 exhibiting a low strain energy channel with a general shape resembling $c_{1}=\beta_{x}^{2}$ across $c_{2}$. The open gaps in the plot are trivial and caused by the data intervals used to produce the data and do not reflect the strain energy which is continuous throughout the $c_{1}-c_{2}-\beta_{x}$ volume. A local minima is highlighted in Figure 9.

Given that the relatively low strain energy region in Figure 9 continues uniformly through values of $c_{2}$, 
this shows that flattening is least influential on the strain energy in this example and so we can analyze the energy landscape using a slice in $\beta_{x^{-}} c_{1}$ space, presented in Figure 10. Upon further analysis, by decreasing the energy limit from $10^{9} \mathrm{Jm}^{-1}$ in Figure 10 (left) to $10^{7} \mathrm{Jm}^{-1}$ in Figure 10 (right), the low strain energy profile becomes visible. A valley, the same as in Figure 9 is observed implying that particular pairings of $c_{1}$ and $\beta_{x}$ values are energetically favorable during deformation of the surface. This observation is intuitive given that coiling up a BRC i.e. decreasing the longitudinal radius of curvature which the term $c_{1}$ simulates, will result in increasing the number of coil turns i.e. increasing the longitudinal subtended angle which the term $\beta_{x}$ represents.

For validation and direct comparison with established straight-BRC models, the initial geometric conditions of the doubly curved-BRC model may be adjusted to converge to straight-BRCs by using infinite longitudinal radius of curvature, $R_{x_{0}} \approx \infty$. This was conducted and presented in Section II with coil radius predictions that agree to approximately $3.75 \%$ with the straight-BRC modeling.

Figure 14 presents the physical result of modeling non-uniform transverse curvature via an alternative computation method using minimization. Adding (ten) higher order cosine terms in Eq. 6 to describe the cross-section shape was found to result in lower energy second stable states with cross-sections that resemble those of Galletly's shell model ${ }^{10}$ where a short "boundary layer" is predicted to form at the edges. For the BRC parameters modeled, the predicted coil radii are in good agreement with the straight-BRC model which predicts $2.22 \mathrm{~cm}$. Increasing the initial longitudinal radius of curvature (labeled $R$ in Figure 14) results in convergence of the doubly curved-BRC model to the straight-BRC result.

\section{Conclusion}

The past methods used to investigate bistability were categorized and chronologically ordered as the simple beam, extended beam, shell and inextensional models. These methods have progressively improved our understanding and analysis of bistability by considering more parameters and effects, however, they do not account for non-zero initial longitudinal curvature and to do so requires an alternative approach. The concept of Gaussian curvature helps define two groups of surfaces; developable and non-developable surfaces or rather, surfaces which can be flattened onto a plane and those that cannot unless stretched or torn. This property of surfaces defines the major departure between preceding BRC research that focussed on developable surfaces and the work presented in this report investigating non-developable surfaces e.g. toroidal-BRCs.

\section{Acknowledgments}

Geoffrey Knott is sponsored by EPSRC and industry, RolaTube Technology Ltd. through industrial CASE.

\section{References}

${ }^{1}$ Iqbal, K., Pellegrino, S., and Daton-Lovett, A., "Bi-Stable Composite Slit Tubes," 1998.

${ }^{2}$ Nettles, A. T., "Basic Mechanics of Laminated Composite Plates," 1994.

${ }^{3}$ Hyer, M. W., Stress Analysis of Fiber-Reinforced Composite Materials, 1998.

${ }^{4}$ Daton-Lovett, A., Compton-Bishop, Q. M., and Curry, R. G., "Deployable Structures Using Bi-Stable Reeled Composites," Smart Structure and Materials, Vol. 3992, 2000, pp. 636-646.

${ }^{5}$ Calladine, C. R., Theory of Shell Structures, Cambridge University Press, 1983.

${ }^{6}$ Iqbal, K. and Pellegrino, S., "AIAA 2000-1385 Bi-Stable Composite Shells," 2000, pp. 1-8.

${ }^{7}$ Galletly, D. A. and Guest, S. D., "Equilibrium and Stability Analysis of Composite Slit Tubes," 2000.

${ }^{8}$ Galletly, D. A., Modelling the Equilibrium and Stability of Slit Tubes, Ph.D. thesis, Cambridge, 2002.

${ }^{9}$ Galletly, D. A. and Guest, S. D., "Bistable composite slit tubes. I. A beam model," International Journal of Solids and Structures, Vol. 41, No. 16-17, Aug. 2004, pp. 4517-4533.

${ }^{10}$ Galletly, D. A. and Guest, S. D., "Bistable composite slit tubes. II. A shell model," International Journal of Solids and Structures, Vol. 41, 2004, pp. 4517-4533.

${ }^{11}$ Guest, S. D. and Pellegrino, S., "Analytical models for bistable cylindrical shells," Proceedings of the Royal Society A: Mathematical, Physical and Engineering Sciences, Vol. 462, No. 2067, March 2006, pp. 839-854.

${ }^{12}$ Gauss, K. F., "General Investigations of Curved Surfaces," Science, Vol. 16, 1902, pp. 902-903.

${ }^{13}$ Mansfield, E. H., The Bending and Stretching of Plates, Second edition, Cambridge University Press, Cambridge, 1989. 